EPiC Series in Computing
Volume 69, 2020, Pages 287-296
Proceedings of 35th International Confer-
ence on Computers and Their Applications

\title{
Integrated adaptive interface design system development of the magnetic separator considering the shapes of the hopper for a powder line
}

\author{
Takashi Ohnishi ${ }^{*{ }^{*} 2^{*} 3}$, and Keiichi Watanuki ${ }^{* 1^{*} 3}$ \\ *1 Advanced Institute of Innovative Technology, Saitama University, Japan \\ 255 Shimo-okubo, Sakura-ku, Saitama-shi, Saitama 338-8570 Japan \\ *2 Magnetec Japan Ltd. \\ 5-521-1, Mikajima, Tokorozawa-shi, Saitama 359-1164 Japan \\ ohnishi@magnetec.co.jp \\ *3 Graduate School of Science and Engineering, Saitama University \\ 255 Shimo-okubo, Sakura-ku, Saitama-shi, Saitama 338-8570 Japan \\ watanuki@mech.saitama-u.ac.jp
}

\begin{abstract}
Foreign metal removal is a key process of quality control in the food and pharmaceutical industries and can possibly be achieved using a magnet separator. Typically, magnetic separators are installed at existing production facilities by remodeling because they have the ability to deal with the problems that arise in production facilities. However, when measuring for remodeling, problems such as measurement error, forgotten measurements, change in location, detail proposal changes, or impossibility to measure occur because of complex, distorted or dented shapes, dimensional inaccuracy, and the surroundings. Additionally, the magnet separators designed to fit an existing production have problems in that the dimensions differ from those of the existing facilities, and deficiency is expected in the performance. To solve these problems using a non-conventional method, we developed an adaptive interface design system that combines high accuracy measurement by means of 3D scan to reproduce the existing production facilities as distorted shape and dented shape by reverse engineering, and the optimized finite element method analysis for magnet field to satisfy an expected performance of the surface flux density, and inspect the shape of the design, dimensions, and performance, using computer aided engineering.
\end{abstract}

\section{Introduction}

Magnetic separators are used to remove foreign metal particles from powders, grains, and liquids. Magnetic separators can only remove magnetized material. Foreign metal particles adhere to the existing processing equipment during sliding and other processes, thus, contaminating the facilities. 
The product-processing equipment are manufactured using austenitic stainless steel, and weak magnetization is often observed following martensite transformation during sliding and collisions. Therefore, we need to install a magnet separator that can remove metal particles of the austenitic stainless steel.

The foreign metal particles are found after the production process in a facility, so installing a magnetic separator has to occur in an existing facility. However, existing production facilities are mostly distorted and dented in shape because operators physically strike them to break powder clogging, as shown in Figure 2; so, it is hard to measure the details of the facility, as shown in Figure 1. We manufacture a trial product, install it, and test the same before the final product is made; this results in deterioration of work efficiency, taking up considerable effort and time.

Moreover, the performances of the magnet field are ambiguity unless it manufactured, and sometime, the product is remanufactured when deficiency in expected performance even if same magnetic arrangement as previous production result.

In order to address these problems, we have investigated and developed an integrated adaptive interface design system with high accuracy measurements for surroundings, taking into account the distorted and dented shapes using 3D scan to reproduce the rough existing production facilities using reverse engineering. Thus, we developed a magnetic separator to find solutions from the constraint condition of magnet material, yoke material, dimension of surroundings, and magnetic circuit, by optimized finite element method analysis for magnet field to inspect shape of design, dimensions, and expected performance for fitting of the hopper and the magnetic separator using computer aided engineering. Then, we made use of the finite element method analysis of magnetic circuit for foreign metal particle removal. Next, we apply reverse engineering for high accuracy measurements, using 3D scan, for inspecting the shape, dimension, and the performance of the optimized magnetic separator, as shown in Figure 3.

\section{Facility as drawing}

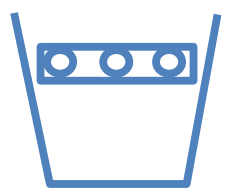

Figure 1: Difference between drawing and real

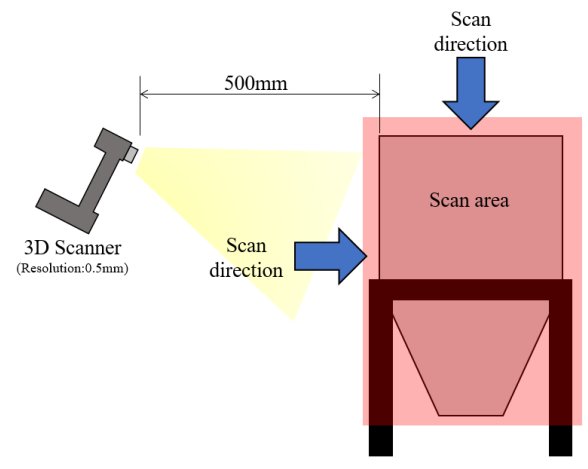

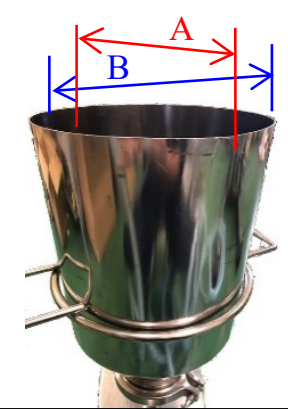

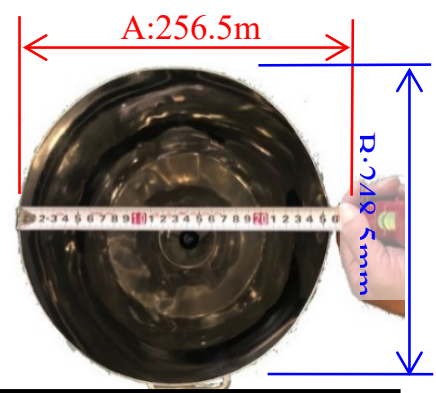

Different dimension for vertical and horizontal

Figure 3: Integrated adaptive interface design system

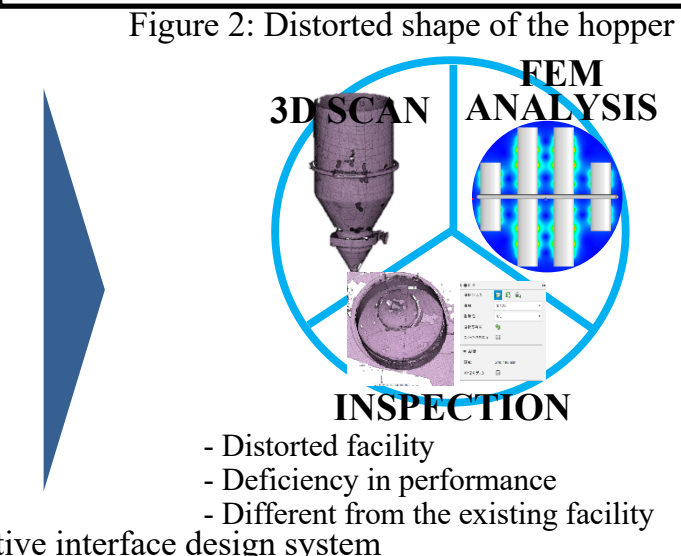




\section{Materials and Methods}

The existing facility, in Magnetec Japan Ltd., in Saitama of Japan, as shown in Figures 4(a)-(c), is measured by a three-dimensional (3D) scanner. The scanner measures the property as a hopper, the shape, the distorted shape, and the dented shape of the facility. (Actual production facilities of our customers are impossible to show by data or photo because of customer's non-disclosure agreement.)

We measure the hopper of the facilities using 3D scan as shown in Figures 4(a)-(c). The scanned data is converted to a 3D model, and the distorted shape and dented shape of the existing hopper are reproduced by reverse engineering, as shown. The magnetic separator is then designed to fit to the existing hopper, as shown in Figure 5. The magnet separator is designed for optimal magnet field performance, and the parameters for magnet material, yoke material, dimension of the magnetic separator, and magnetic circuit, are set up for finite element analysis.

The device used to capture the 3D measurements is the "GO! SCAN 50 of AMETEK Inc.," as shown in Figure 6. The scanned data is converted to 3D modeling by computer software, "VXelements" of AMETEK Inc. The computer software for analyzing the system with finite element analysis is "ANSYS Electromagnet Suite 19.1[1]", and the computer is a "DELL PRECISION TOWER 7810". The scheme is "3D Maxwell's equations", mesh size is $4.82 \mathrm{~mm}$, and solver is "Magnetostatic", as shown in Figures 7(a)-(d) and Table 1. The mesh size is determined to be $4.82 \mathrm{~mm}$ for deficiency in performance of the machine with reference [2] because mesh size is $2.5 \mathrm{~mm}$ in reference [3]. However, the quantity of magnet pieces in the magnetic separator is increased.

The 3D scanner measures not only the hopper as subject, but also its distance to the surroundings. The finite element analysis set up parameters, such as the residual magnetic flux density, relative permeability, and magnetic coercivity, of four magnet characteristics- N38, N40, N48, and ferrite, as shown in Table 2; then, the magnet characteristic, which satisfied the condition for surface flux density of 1.0 Tesla, is selected. Thus, the magnet characteristic of the magnet piece is found by pareto optimal solution from the results of the finite element method analysis simulation.

Thereby, the 3D model of the magnet separator is inspected for fitting and rattling into the hopper, using 3D computer-aided design as shown in Figures 8(a)-(c).

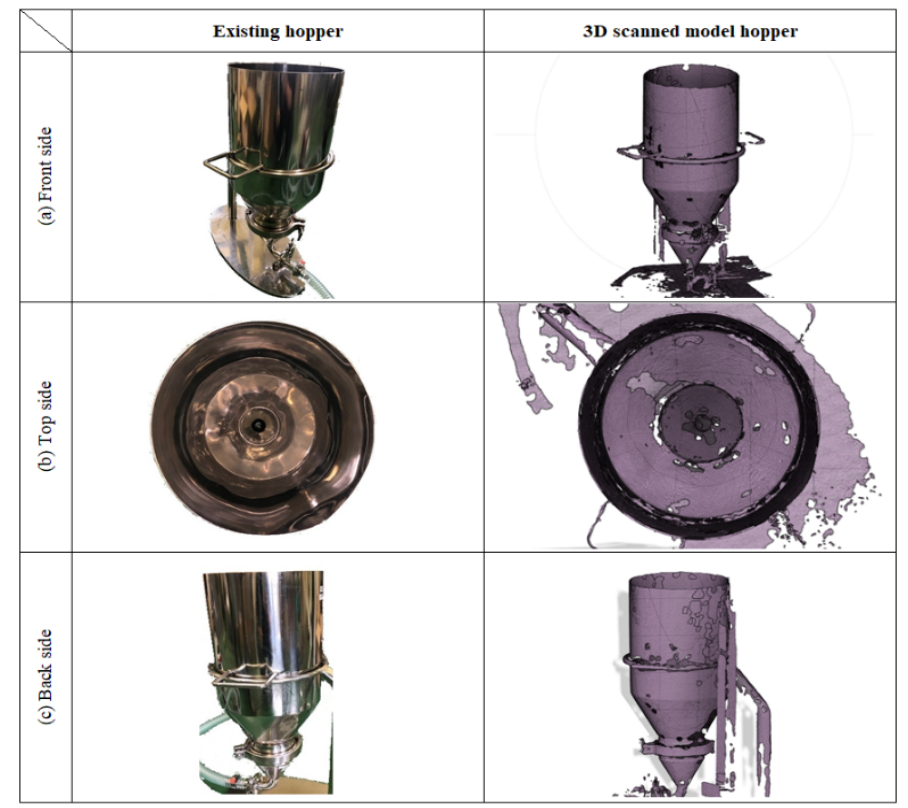

Figure 4: The existing and the 3D scanned hopper 

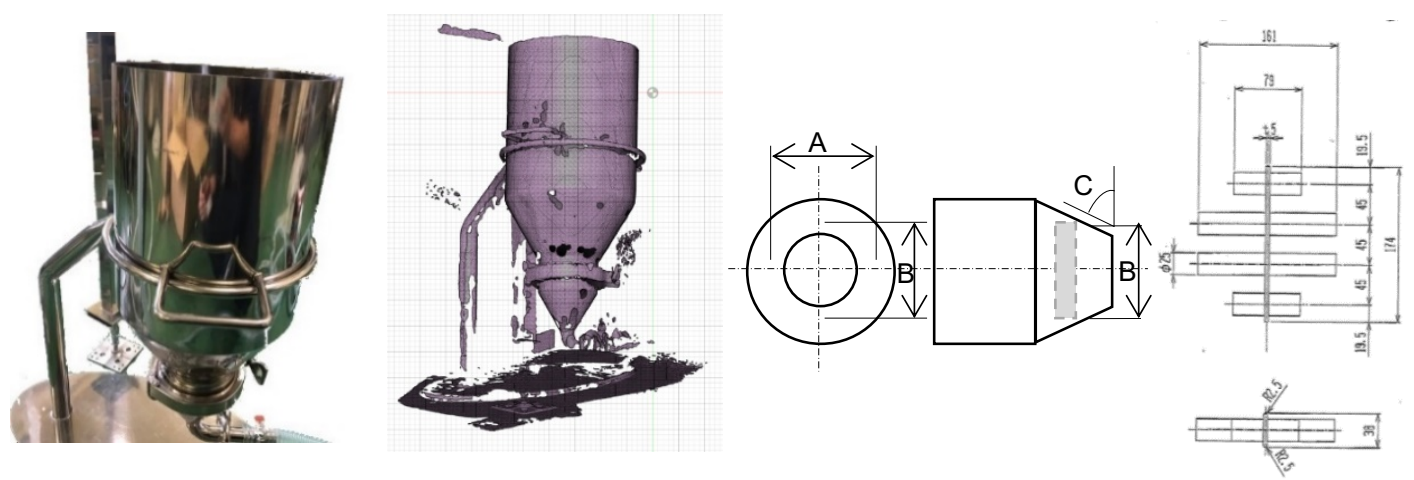

Scan existing hopper

Make 3D model

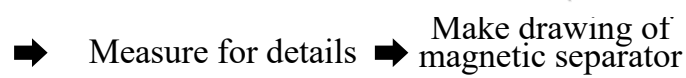

Figure 5: Design flow of the integrated interface design system

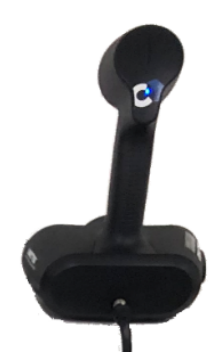

(a) 3D scanner

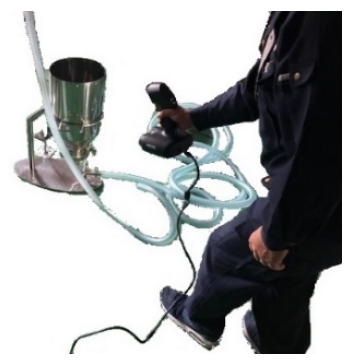

(b) Scanning for the existing hopper

Figure 6: Scanning for the hopper by 3D scanner

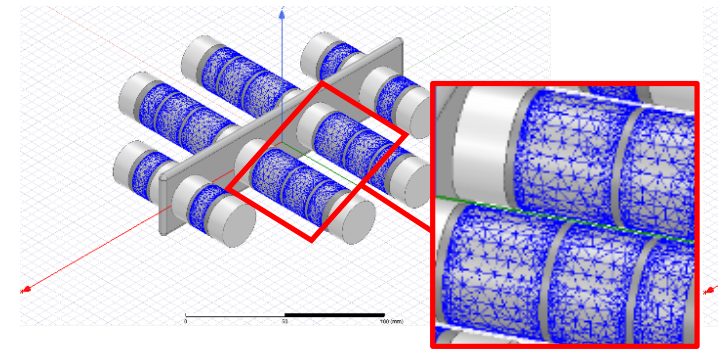

(a) Mesh analysis of N38 characteristic

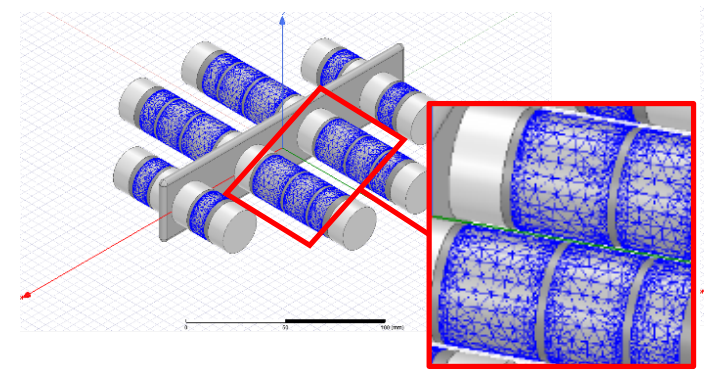

(c) Mesh analysis of N48 characteristic

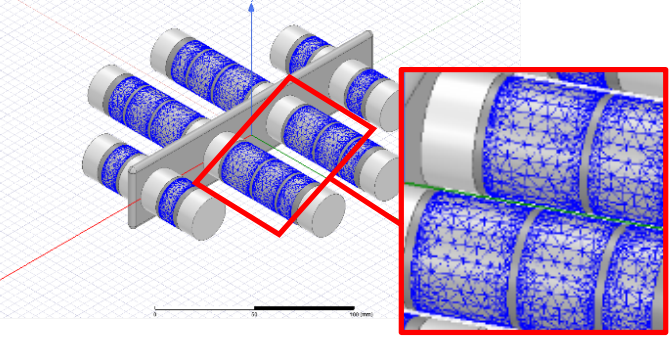

(b) Mesh analysis of N40 characteristic

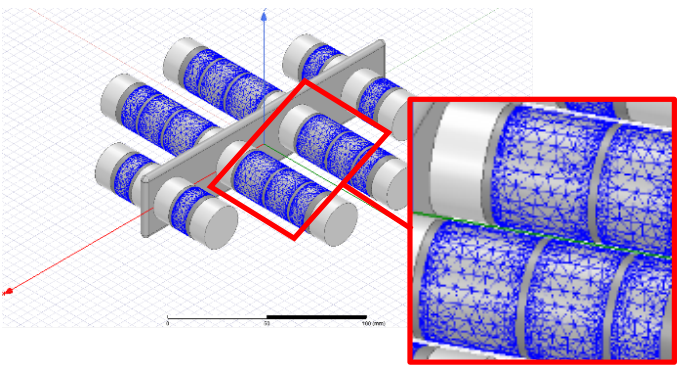

(d) Mesh analysis of ferrite characteristic

Figure 7: Mesh analysis of the magnet separators 
Table 1: Specifications of FEM analysis

\begin{tabular}{|c|c|c|c|c|c|}
\hline Analy & $\begin{array}{l}\text { finite eleme } \\
\text { vice }\end{array}$ & $\begin{array}{l}\text { nethod: ANSYS } \\
\text { : DELL PRI }\end{array}$ & $\begin{array}{l}\text { ctromagnet } \\
\text { SION TOW }\end{array}$ & $\begin{array}{l}\text { ite } 19.1 \\
10\end{array}$ & \\
\hline 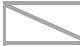 & Mesh size & Tetrahedra & Real time & Solver & Scheme \\
\hline N38 & $4.82 \mathrm{~mm}$ & 848251 & 1:22:39 & Magnetostatic & Three-dimensional Maxwell's equations \\
\hline N40 & $4.82 \mathrm{~mm}$ & 848680 & 1:23:02 & Magnetostatic & Three-dimensional Maxwell's equations \\
\hline N48 & $4.82 \mathrm{~mm}$ & 848577 & 1:21:45 & Magnetostatic & Three-dimensional Maxwell's equations \\
\hline Ferrite & $4.82 \mathrm{~mm}$ & 1103285 & $0: 30: 23$ & Magnetostatic & Three-dimensional Maxwell's equations \\
\hline
\end{tabular}

Table 2: Parameters of the magnet characteristic

\begin{tabular}{|l|l||r|r|r|}
\hline \multicolumn{2}{|c|}{ Name } & Relative permeability & Magnetic coercivity & Composition \\
\hline \multirow{2}{*}{ N38 } & Magnet & 1.01050757518664 & $-979.65 \mathrm{kA} / \mathrm{m}$ & Solid \\
\cline { 2 - 6 } & Yoke & B-H Curve & $0 \mathrm{~A} / \mathrm{m}$ & Solid \\
\hline \multirow{2}{*}{ N40 } & Magnet & 1.02271873586037 & $-992.93 \mathrm{kA} / \mathrm{m}$ & Solid \\
\cline { 2 - 6 } & Yoke & B-H Curve & $0 \mathrm{~A} / \mathrm{m}$ & Solid \\
\hline \multirow{2}{*}{ N48 } & Magnet & 1.03880530314316 & $-13.426 \mathrm{kOe}$ & Solid \\
\cline { 2 - 6 } & Yoke & B-H Curve & $0 \mathrm{~A} / \mathrm{m}$ & Solid \\
\hline \multirow{2}{*}{ Ferrite } & Magnet & 1.31277962794766 & $-2.9727 \mathrm{kOe}$ & Solid \\
\cline { 2 - 6 } & Yoke & B-H Curve & $0 \mathrm{~A} / \mathrm{m}$ & Solid \\
\hline
\end{tabular}

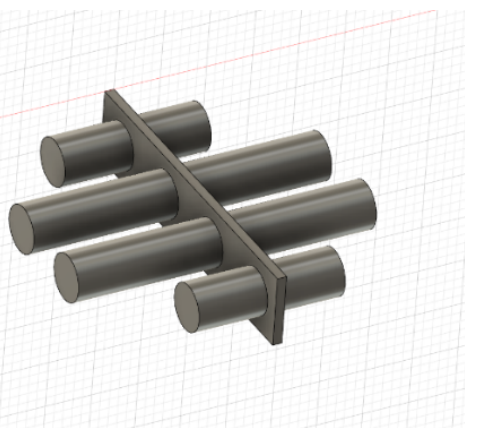

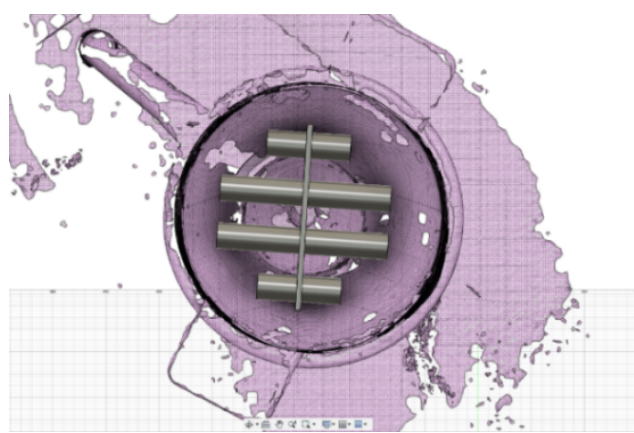

(b) Installation the magnet separator to the hopper

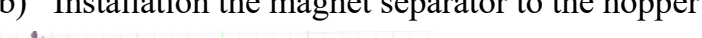

\section{(a) 3D model of the magnetic separator}

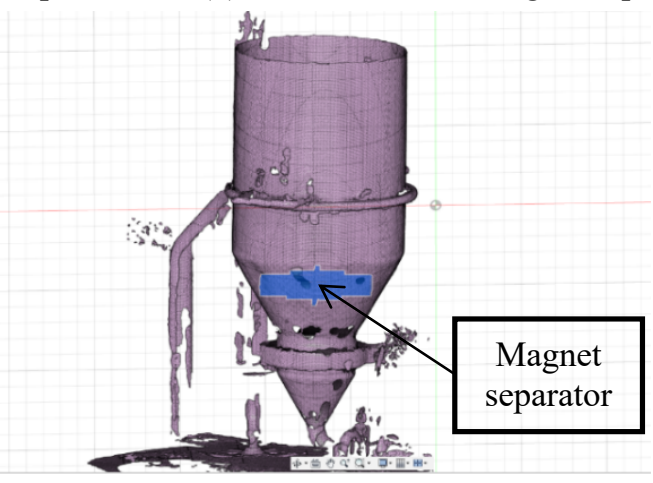

(c) Inspecting for fitting and rattling

Figure 8: Inspection for installation by CAE 


\section{Result}

The high-accuracy 3D scan for the hopper and the finite element analysis method for the magnetic separator were inspected for shape, dimension, and performance of the magnetic separator, prevented failed product and the loss of material, and validated for traceability of design qualification. At first, the $3 \mathrm{D}$ scan is measured for shape and dimension of the existing hopper, and also for the distorted and dented shapes, as shown in Figure 9.

The verification process due to measurement error is ineffective because the conventional method of single dimensional measurement, using ruler, tape measure, and caliper, depends on the reverse engineering. The measurement accuracy of the 3D scan is high compared with single dimensional measurement because the accuracy of the 3D scan is $0.1 \mathrm{~mm}$, as shown in Table 3 and Figure 10. Additionally, the 3D model of the magnetic separator was designed for perfect fit to the scanned hopper with the same shape and dimension, as shown in Figures 11(a)-(d). The magnet characteristic is selected from four characteristics using the finite element method analysis, as shown in Figures 12(a)-(d) and considered for the marketability of cost. The magnet characteristics used are N38, N40, $\mathrm{N} 48$, and ferrite. These magnet characteristics have differences in residual magnetic flux density, relative permeability, and magnetic coercivity. The threshold of the surface flux density is 1.0 Tesla and the magnetic characteristics are selected to satisfy for the value of 1.0 Tesla.

To find the solution by pareto optimal solution, three characteristics meet the surface flux density of 1.0 Tesla. N38 was selected because of cost consideration, as shown in Figure 13. From these findings, the actual magnetic separator is manufactured and then inspected for shape, dimension, fitting, and rattling into the existing hopper, thus validating and ensuring the traceability of the design qualification, as shown in Table 4.
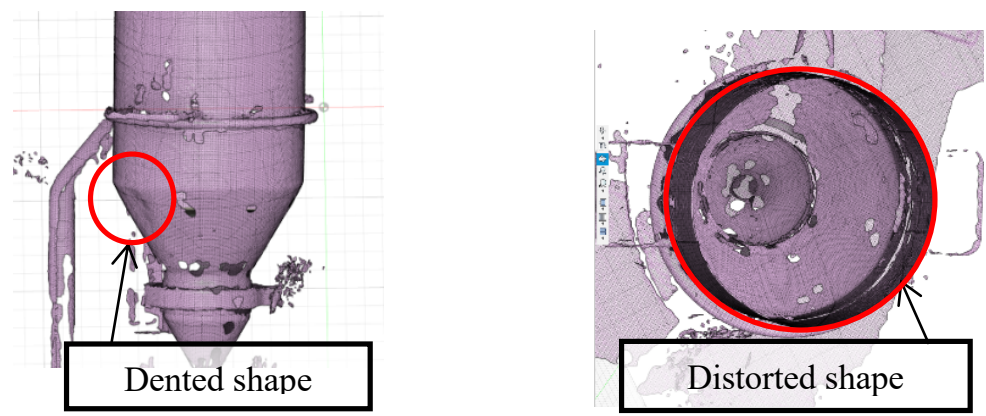

Figure 9: Re-product of the existing hopper by 3D scan

Table 3: Specifications of "GO! SCAN 50"

\begin{tabular}{|l|l|}
\hline \multicolumn{2}{|c|}{ Specifications of Go!SCAN 50 } \\
\hline Weight & $950 \mathrm{~g}$ \\
\hline Dimension & $150 \times 171 \times 251 \mathrm{~mm}$ \\
\hline Scan speed & $550,000 \mathrm{Vertex} / \mathrm{sec}$ \\
\hline Scan area & $380 \times 380 \mathrm{~mm}$ \\
\hline Light source & LED white light \\
\hline Resolution & $0.500 \mathrm{~mm}$ \\
\hline Accuracy & Max0.100 mm \\
\hline Standoff distance & $400 \mathrm{~mm}$ \\
\hline Depth of field & $250 \mathrm{~mm}$ \\
\hline Texture resolution & $50 \sim 150 \mathrm{DPI}$ \\
\hline Texture color & $24 \mathrm{bit}$ \\
\hline Software & VXelements \\
\hline
\end{tabular}



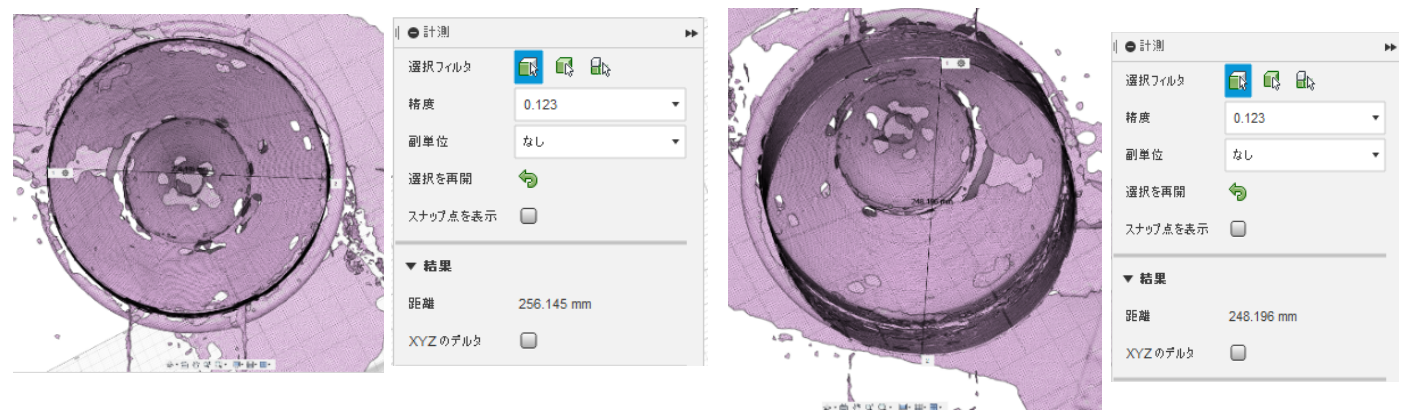

\begin{tabular}{lll} 
& 1D measurement & 3D scan \\
Vertical & $248.5 \mathrm{~mm}$ & $248.196 \mathrm{~mm}$ \\
Horizontal & $256.5 \mathrm{~mm}$ & $256.145 \mathrm{~mm}$ \\
& & \\
\hline
\end{tabular}

Figure 10: Accuracy of 3D scan

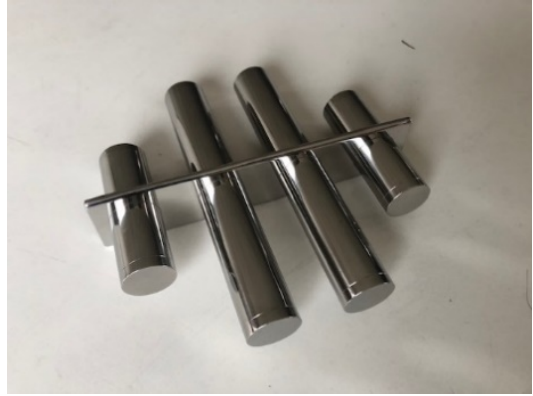

(a) Magnetic separator based on 3D model

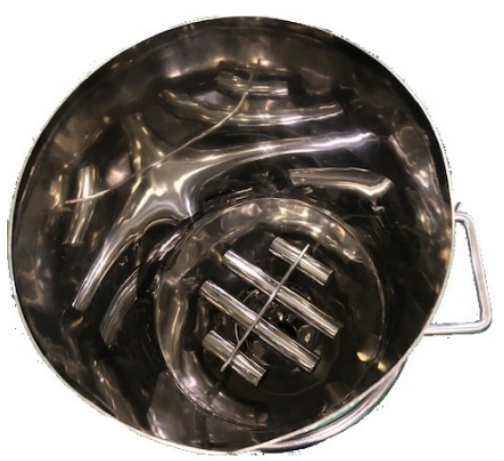

(c) Installation the magnet separator to the hopper

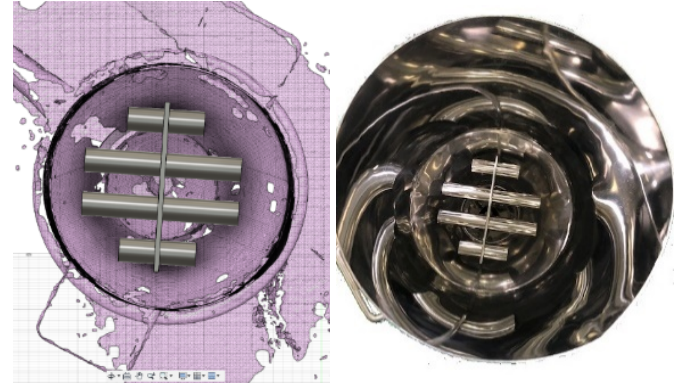

(b) Compare with CAE and actual installation

Figure 11: The magnetic separator fit to the existing hopper 
Integrated interface design system construction of the magnet separator in ... Ohnishi and Watanuki
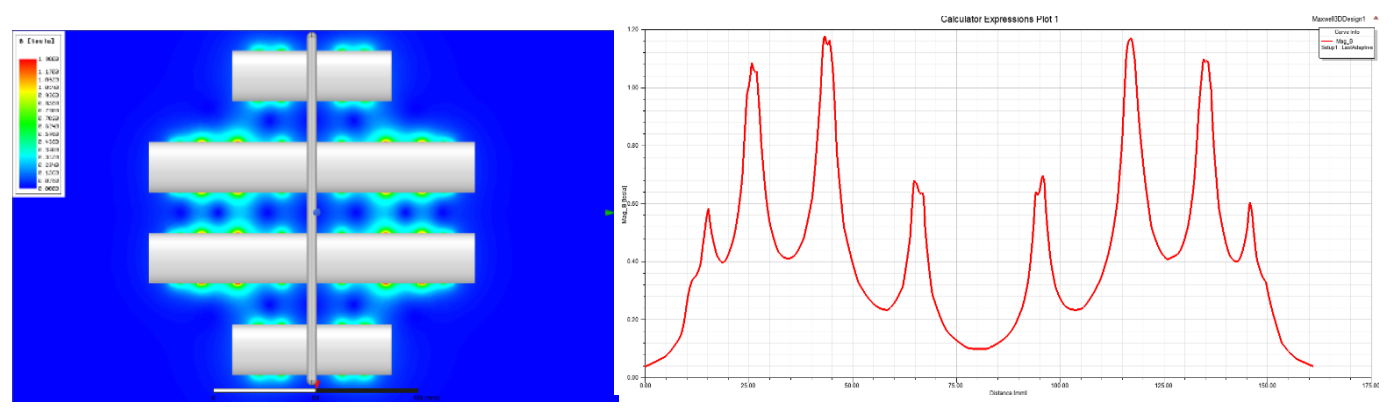

(a) Finite element method analysis for N38
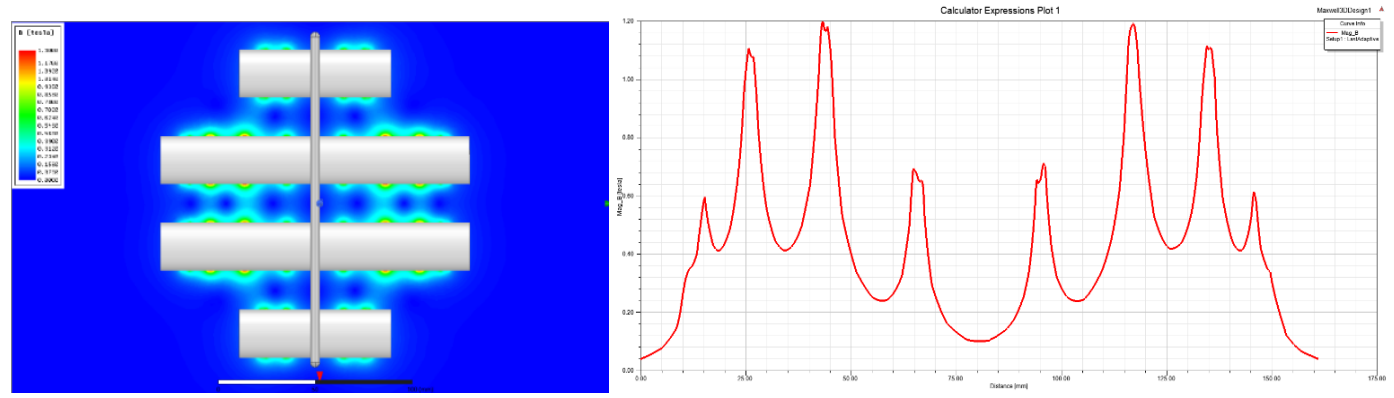

(b) Finite element method analysis for N40
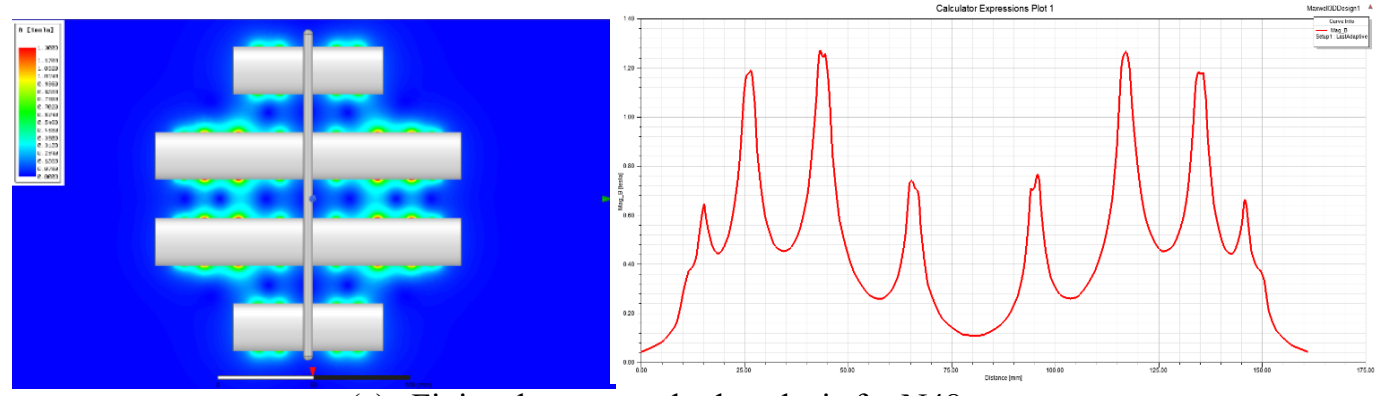

(c) Finite element method analysis for N48
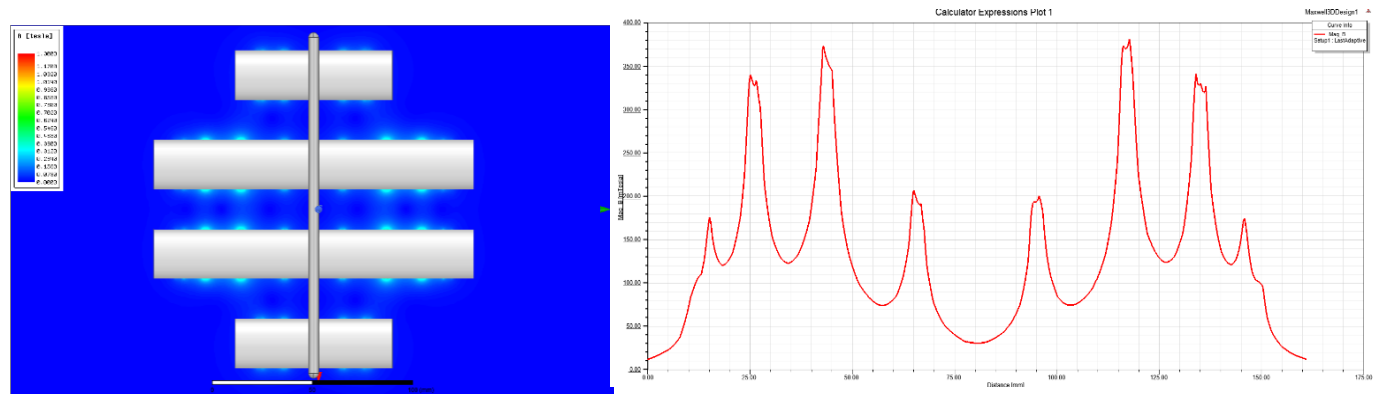

(d) Finite element method analysis for ferrite

Figure 12: Magnetic field and surface flux density 


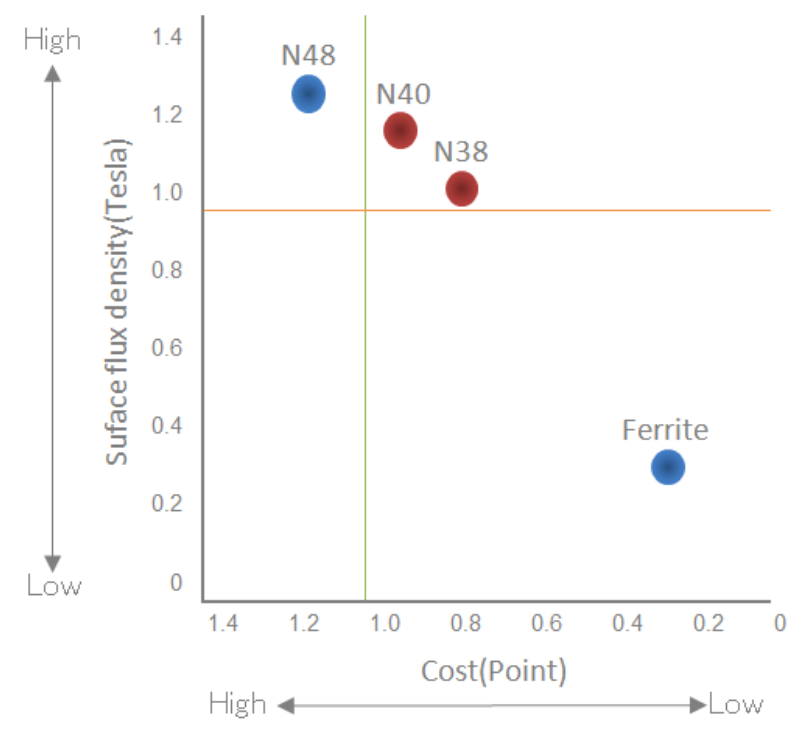

Figure 13: Pareto optimization for magnet

Table 4: Traceability of the design qualification

\begin{tabular}{|l|l|l|l|}
\hline Item & Criteria & Result & Accept \\
\hline Shape & Round type & Round type & Accepted \\
\hline Dimension & Conform to drawing & Conformed to drawing & Accepted \\
\hline Surface flux density & 1.0 Tesla & 1.0135 Tesla & Accepted \\
\hline Installation & Exactly fit & Exactly fitted & Accepted \\
\hline
\end{tabular}

\section{Conclusions}

In this paper, we developed a solution for contamination of products at production facilities. During processing, foreign metal particles of austenitic stainless steel were found and removed by a magnetic separator with magnetic force. We developed a non-conventional method an integrated adaptive interface design system to solve this problem that combines high accuracy measurement by 3D scan to reproduce the rough existing production facilities and took into consideration the distorted shape and dented shape, using reverse engineering, and the optimized finite element method analysis for magnet field to satisfy an expected performance of the surface flux density, and inspect shape of design, dimensions, and performance by computer aided engineering.

Previously, the existing production facilities were measured using single dimensional measurement that employed a ruler, tape measure, and caliper; moreover, the product of the magnet separator was manufactured from same magnetic arrangement as that of the previous production result, so the magnetic separator did not fit or rattle to the existing production facilities. However, these problems are solved by the integrated adaptive interface design system. Also, the system will help the sheet metal working, pipe working, machining, repair, and alteration of the facilities. The scanner is high accuracy 3D measurement to measure distorted shape and dented shape, which compare with single 
dimensional measurement as measurement error and impossible to measure, for re-product of reverse engineering by 3D scan, so it can investigate the cause of; tracing something back to its origin when problem occurred. The finite element method analysis is possible for validation and estimation of performance of the magnetic separator before manufacture, as the trial products and off-the-grade products can be substantially reduced.

Thus, the integrated adaptive interface design system of manufacture for optimized magnetic separator was developed using 3D scan and the finite element method analysis was used to inspect the dimension, shape, and performance of the magnetic separator and to prevent failed product and the loss of material, using computer aided engineering. This is a significant development for visualization of the magnet field, surface flux density, and the magnet area, for foreign metal removal, in particular, for designing an optimized magnetic separator in reverse-engineered existing facilities.

Thus far, the previous production results were investigated and a trial product was installed before manufacturing the magnetic separator, but it will be eliminated those works. Thus, there are merits in incorporating the proposed system for reducing the cost of the material, labor, and operation duration.

Hereafter, the integrated adaptive interface system will be able to manufacture the optimized magnetic separator to help improve the working efficiency, even if it is impossible to design because an existing drawing is missing or the existing facilities are impossible to measure.

\section{References}

[1] S. Farinon, "Magnet design and optimization: The infn-Genova experience using ansys," Cryogenics, 47:577-582, 2007.

[2] E.V. Karpenko, N.A. Kolbasina, and T.V. Ershova, "Modeling of three - dimensional magnetostatic analysis in the ansys workbench," elib.sfu-kras.ru, 2011.

[3] T. Ohnishi, T. Okamoto, and K. Watanuki, "Comparison of Metal-Contamination Removal Rates When Using Three Magnets of Different Shapes," International Journal of Computers and Their Applications, 26(3):108-119, 2019. 